Scientific Visualization, 2020, volume 12, number 2, pages 1 - 8, DOI: 10.26583/sv.12.2.01

\title{
Visualization of Multidimensional Hypersurface Extrema by Lumigraph
}

\author{
Eugene Popovi,A, Tatyana Popova2,B, Anatoly Batiukov3,A, Natalja Vogt4,C,D \\ A Nizhegorodsky State Architectural and Civil Engineering University \\ B National Research University Higher School of Economics \\ C University of Ulm (Germany) \\ D Lomonosov Moscow State University \\ ${ }^{1}$ ORCID: 0ooo-0002-3058-2369, popov eugene@list.ru \\ 2 ORCID: 0000-0002-1351-222X, tatpop@list.ru \\ 3 ORCID: 0000-0002-1050-1883, absterno@gmail.com \\ 4 ORCID: 0000-0001-7190-5702, natalja.vogt@uni-ulm.de
}

\begin{abstract}
The paper describes a method for a multidimensional hypersurface and its extrema visualization by Lumigraph model. The paper shows the shortcomings of the classic Lumigraph and formulates the reasons for this. To overcome these shortcomings the authors introduce the additional screen concept. The rules for constructing an image on this screen are described. The final image makes the entire hypersurface and zones of its extrema clearly visible.
\end{abstract}

Keywords: Multidimensional visualization, Hypersurface extrema, Lumigraph.

\section{Introduction}

The basic feature of the information is its multidimensionality. Due to this fact information often loses its clearness and one cannot represent the data in visual form by standard visualization means such as plots, graphs and diagrams [1-3]. This problem frequently occurs in different areas of human activity. For example, in work [4] the scholar attempted to systematize and briefly describe some types of visualization problems arising from processing the results of a generalized mathematical experiment in computational gas dynamics. The author also marshalled the emerging tasks of visual presentation of multidimensional numerical output data. The development of procedures for analyzing the results of a generalized computational experiment presented in the form of a multidimensional data volume is considered in [5].

In chemistry understanding and predicting the properties of chemical compounds are of paramount importance both from technological and academic points of view. Quantum chemical computational methods become useful tools for solving some chemical problems as well as for studying the reaction mechanism prior to difficult, expensive or sometimes impossible experiments. Usually, the problem converges to the extrema set finding of the Potential Energy Surface (PES) related to the so called "molecular conformal landscape" [6], [7]. In the majority of cases PES has a multidimensional character. In this paper PES depends on four independent variables. We will call it $5 \mathrm{D}$ hypersurface. The problem of multidimensional PES visualization is very relevant and complex.

There are numerous visualization approaches and a good number of visual taxonomies [8]. Looking at them the following stand out as high dimensional visualizations: $2 \mathrm{D}$ and $3 \mathrm{D}$ scatterplots; Matrix of scatterplots; Heat maps; Height maps; Table lens; Survey plots; Iconographic displays; Dimensional stacking (general logic diagrams); Parallel coordi- 
nates; Line graph, multiple line graph; Pixel techniques, circle segments; Multidimensional scaling and Sammon plots; Polar charts; RadViz; PolyViz; Principal component and principal curve analysis; Grand Tours; Projection pursuit; Kohonen selforganizing maps and many others. However, the analysis in work [6] has shown that none of these is suitable enough for PES visualization.

\section{Visualization 4D Data by Lumigraph}

There is a possibility to represent a $5 \mathrm{D}$ hypersurface by function of four independent variables with energy level represented by color and apply the model called Lumigraph. The usual two-plane parameterized light field (2PP) extended with object geometry for the reconstruction process was described by Görtler [9] who coined the term Lumigraph. It is usually applied to stress the fact that proxy geometry is required and used for light field reconstruction. It is a subset of the complete plenoptic function that describes the flow of light in all positions and in all directions. The two-plane setup is a global parameterization which describes a ray in space with the intersection points on two parallel planes. Each intersection point is $2 \mathrm{D}$ and this leads to four sampling parameters. In our case this kind of ray can be considered as a $4 \mathrm{D}$ point. A point color considered as an additional parameter transforms this point into a $5 \mathrm{D}$ point.

The main idea can be explained by the example of 2D analogue of Lumigraph (see Fig. 1). Let there be two parallel axes $X_{1}$ and $X_{2}$ related to two independent variables. Then the line between two points at these axes is adequate to $2 \mathrm{D}$ point with coordinates $\left(x_{1}, x_{2}\right)$ in Cartesian space.

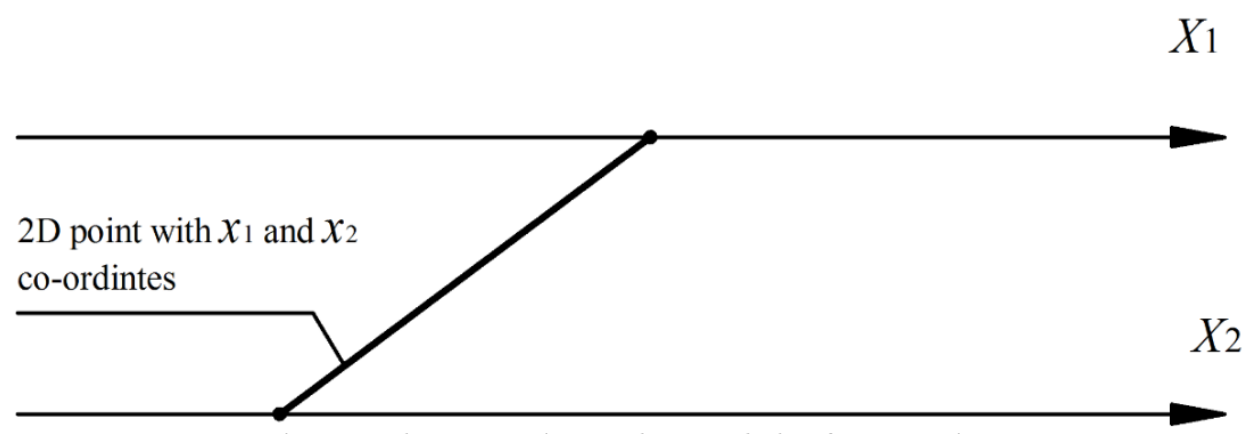

Fig. 1. The Lumigraph model of 2D point

Let us now have a $2 \mathrm{D}$ quadratic matrix of $n \times n$ points and apply this scheme to visualize it as shown in Fig. 2. We can obtain the net of $n^{2}$ lines that can be named 2D Lumigraph representation of $3 \mathrm{D}$ tabulated function $z=f\left(x_{1}, x_{2}\right)$ of two independent variables. If we assign an appropriate color to each point related to $z$ value we can have a complete Lumigraph representation of that function which can be visualized by colored plot.

$X_{1}$

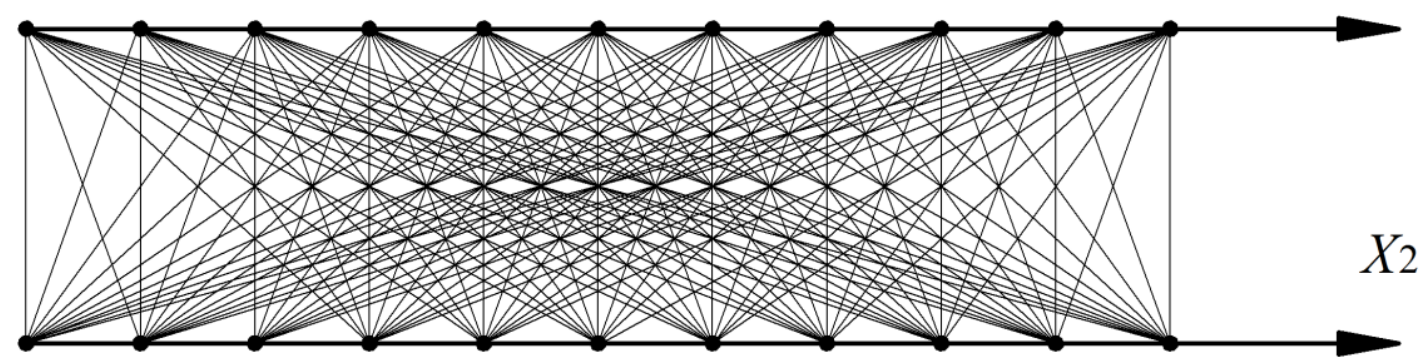

Fig. 2. The set of 2D points in Lumigraph 
If we expand this idea to the case of four independent variables, the point can be represented by the line shown in Fig. 3 where two ending points of it have two pairs of variables (D3, D1 and D6, D5) associated with a corresponding plane.

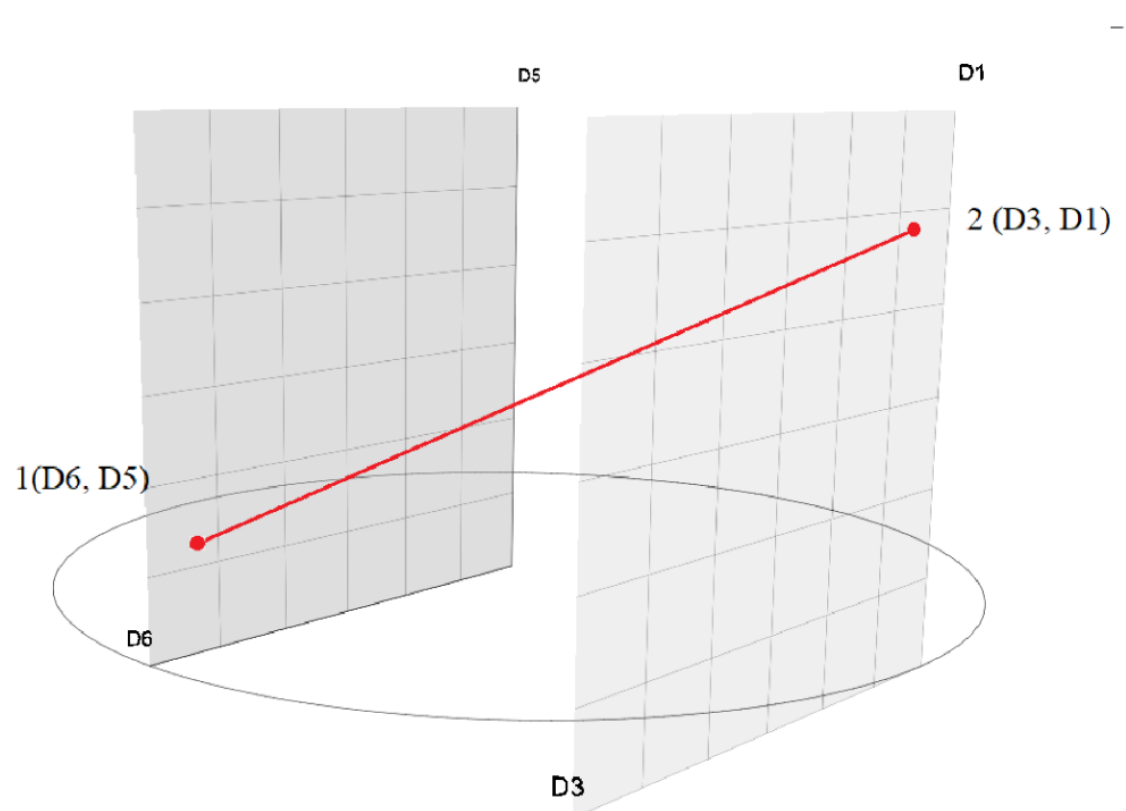

Fig. 3. The point depending on four variables

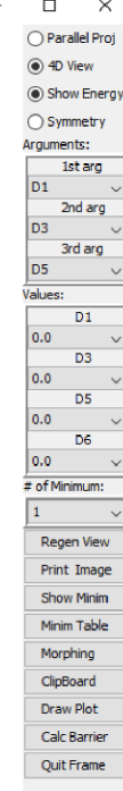

uit Frame

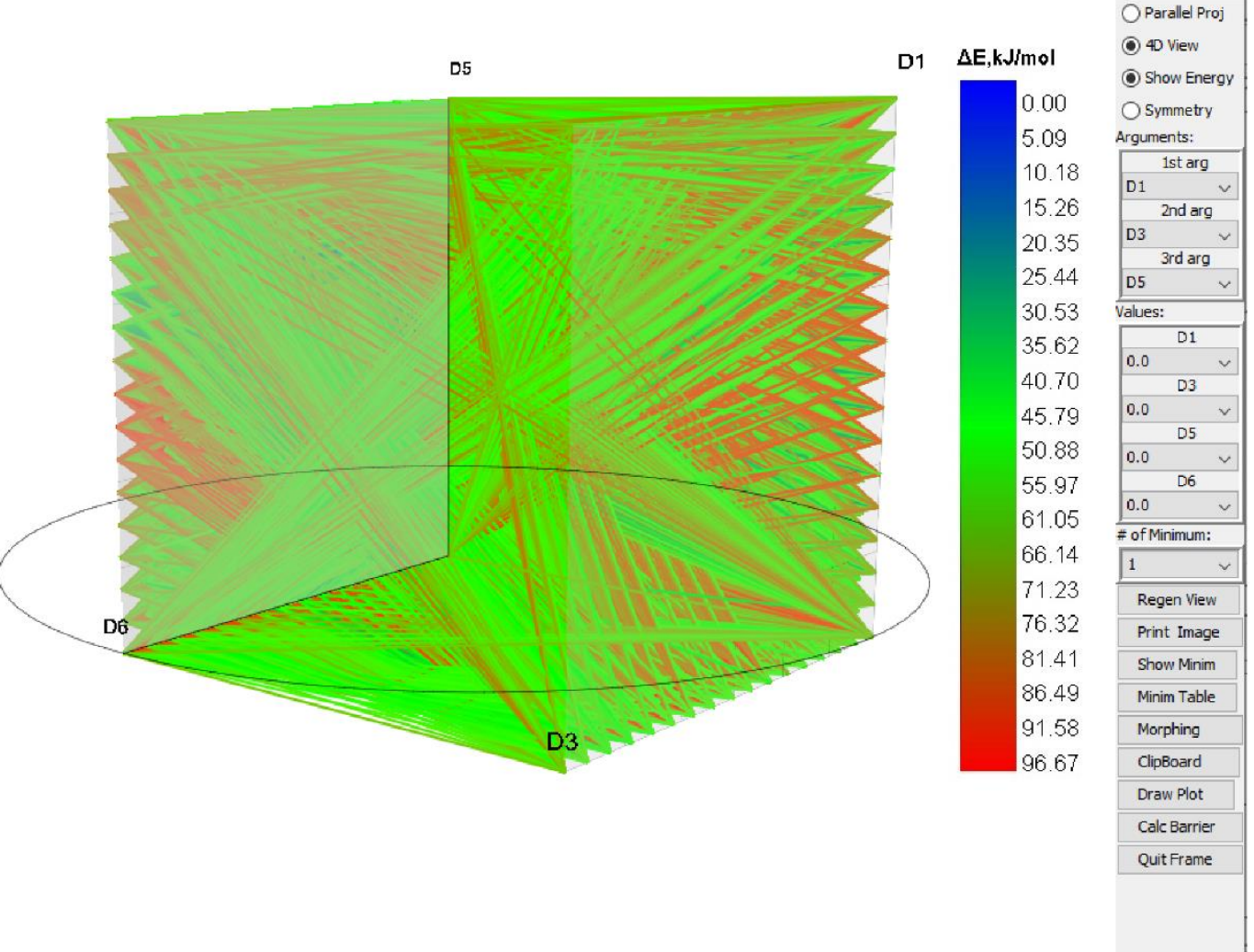

Fig. 4. Tabulated function of four variables modelled by Lumigraph

In geometry, any surface is considered as an infinite point set. However, in most applications, the function of multidimensional surface is presented in the form of finite fourdimensional tables. It means that graphs of such function can be modelled as a finite set of $4 \mathrm{D}$ points only. Lumigraph is a convenient and simple model to visualize the function of 
four variables as seen in Fig. 4 where the function values are displayed in colored form. Usually, the initial $4 \mathrm{D}$ function table has $n$ rows and columns at each dimension, so the number of $4 \mathrm{D}$ points in Lumigraph is equal to $n^{4}$. Though such representation is very simple, obvious and convenient it is not clear enough for perception. The areas of the function extrema can hardly be seen. With this in mind, we have come to conclusion that there is a need to find a way to improve Lumigraph visualization approach.

\section{Mapping of $5 \mathrm{D}$ surface image to additional screen}

Further development of the described approach may be the mapping of Lumigraph to 2D plot. The shape of $2 \mathrm{D}$ plot should be efficient enough so that the entire surface is visible to the user. The main idea here is to place the third plane parallel to two initial ones inside the Lumigraph so as to find the points of intersection of line segments with it. We called it the additional screen concept. One can see the complete $2 \mathrm{D}$ mapping of the whole $5 \mathrm{D}$ hypersurface in the form of raster image (see Fig. 5 where 4D points are hidden).

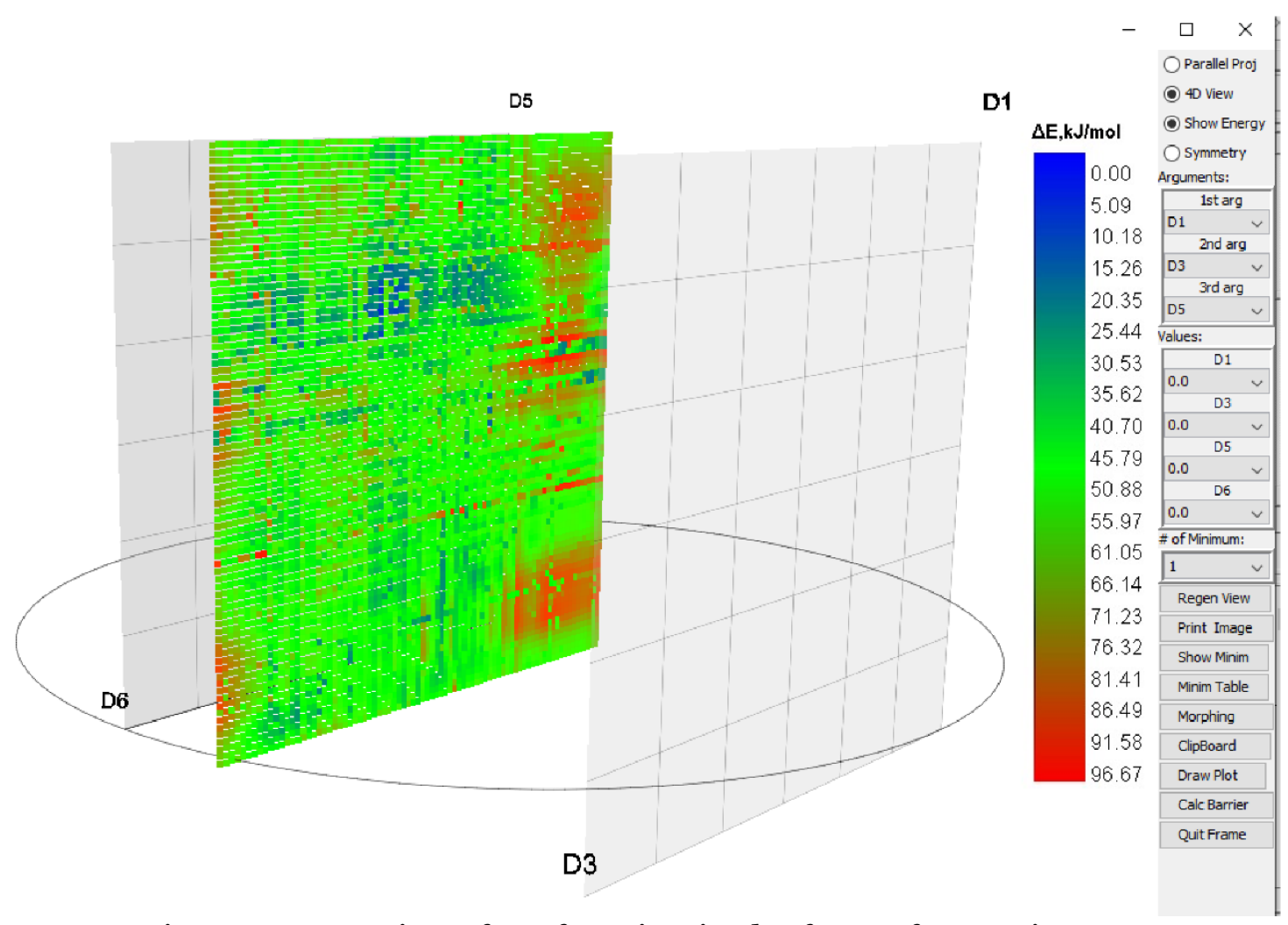

Fig. 5. 2D mapping of 5D function in the form of raster image

The entire extrema hypersurface zones are clearly seen in this image (minima zones are in blue; maxima are in red). The previously described ineffective (see works [6], [7]) method for displaying hypersurface minima (see Fig 6) can now be significantly improved by combining the two models shown in Fig. 5 and Fig. 6. 


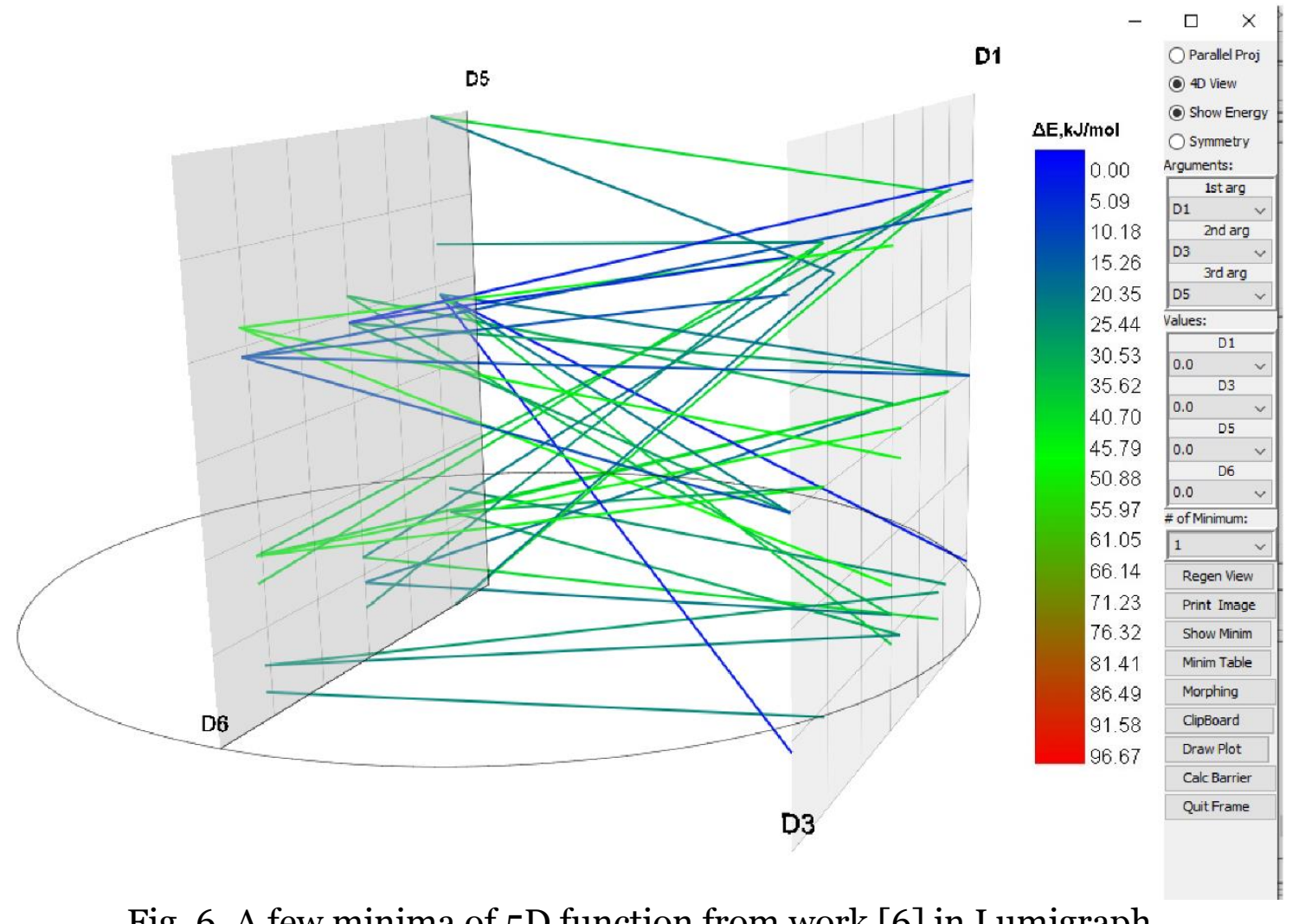

Fig. 6. A few minima of 5D function from work [6] in Lumigraph

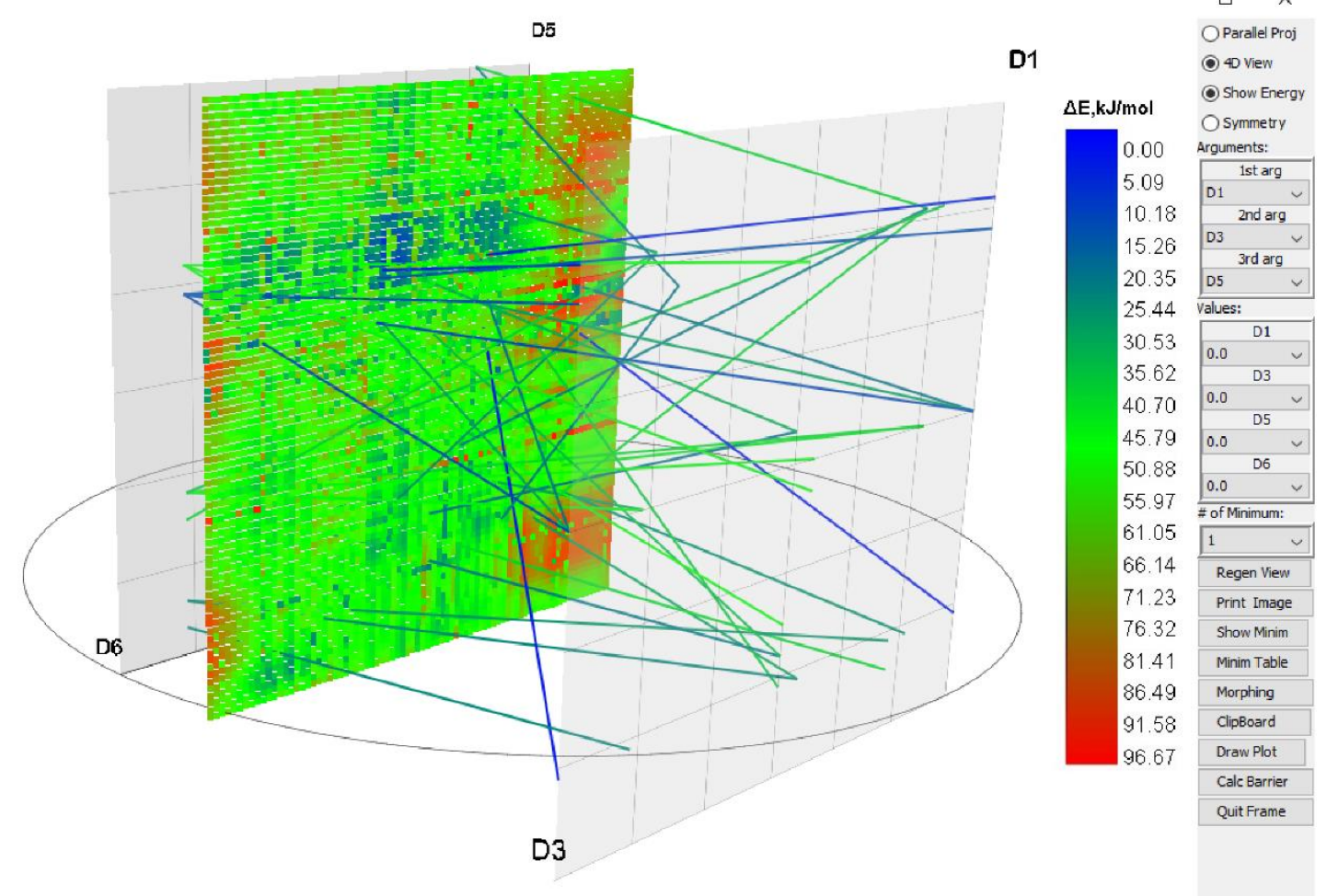

Fig. 7. 5D function minima from work [6] together with 2D image

One can notice that the additional screen is placed at different distances from Lumigraph planes. This is due to the need to increase the number of pixels in the raster image. As can be seen in Fig. 1, the image contains the smallest number of pixels when the mapping plane is located at the middle of two original Lumigraph planes. We placed the additional screen at $0.25 L$ distance from the far Lumigraph plane (see Fig. 5 , here $L$ - the whole distance between the Lumigraph planes). We chose this distance experimentally since the goal was to 
obtain the hypersurface image of a better quality. To give more specific recommendations additional studies of this issue are required.

We also attempted to modify $2 \mathrm{D}$ image by adding the real $5 \mathrm{D}$ function values to Lumigraph as shown in Fig. 8. It consists of several steps. First, we normalize the function value in its min - max range so that it has value in range $(0 ; 1)$. Then we shift the intersection point vertically at the distance $A B$ (see Fig. 8 ) in order to obtain distance $B C$ equal to real function value in $4 \mathrm{D}$ point $1-2$.

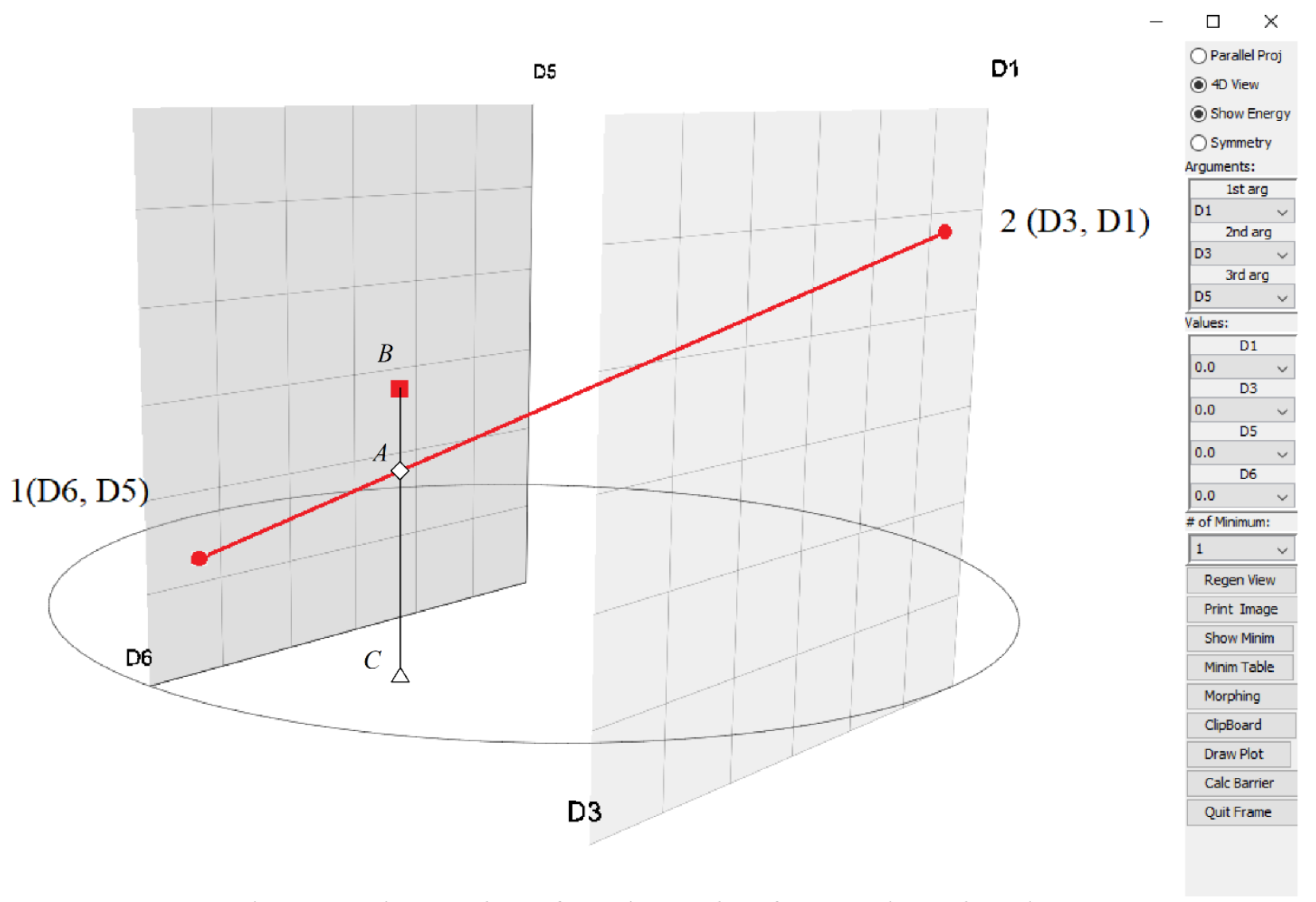

Fig. 8. Assign real $5 \mathrm{D}$ function value for Lumigraph point

After applying this scheme to Lumigraph 2D the image of $5 \mathrm{D}$ function takes the form shown in Fig. 9. It stands to reason that such visualization produces a very demonstrative view of the hypersurface together with its global and local extrema. Only one circumstance remains unclear in Fig. 9, i.e. how all extrema are related to the value of their variables. This circumstance needs additional visual clarification. 


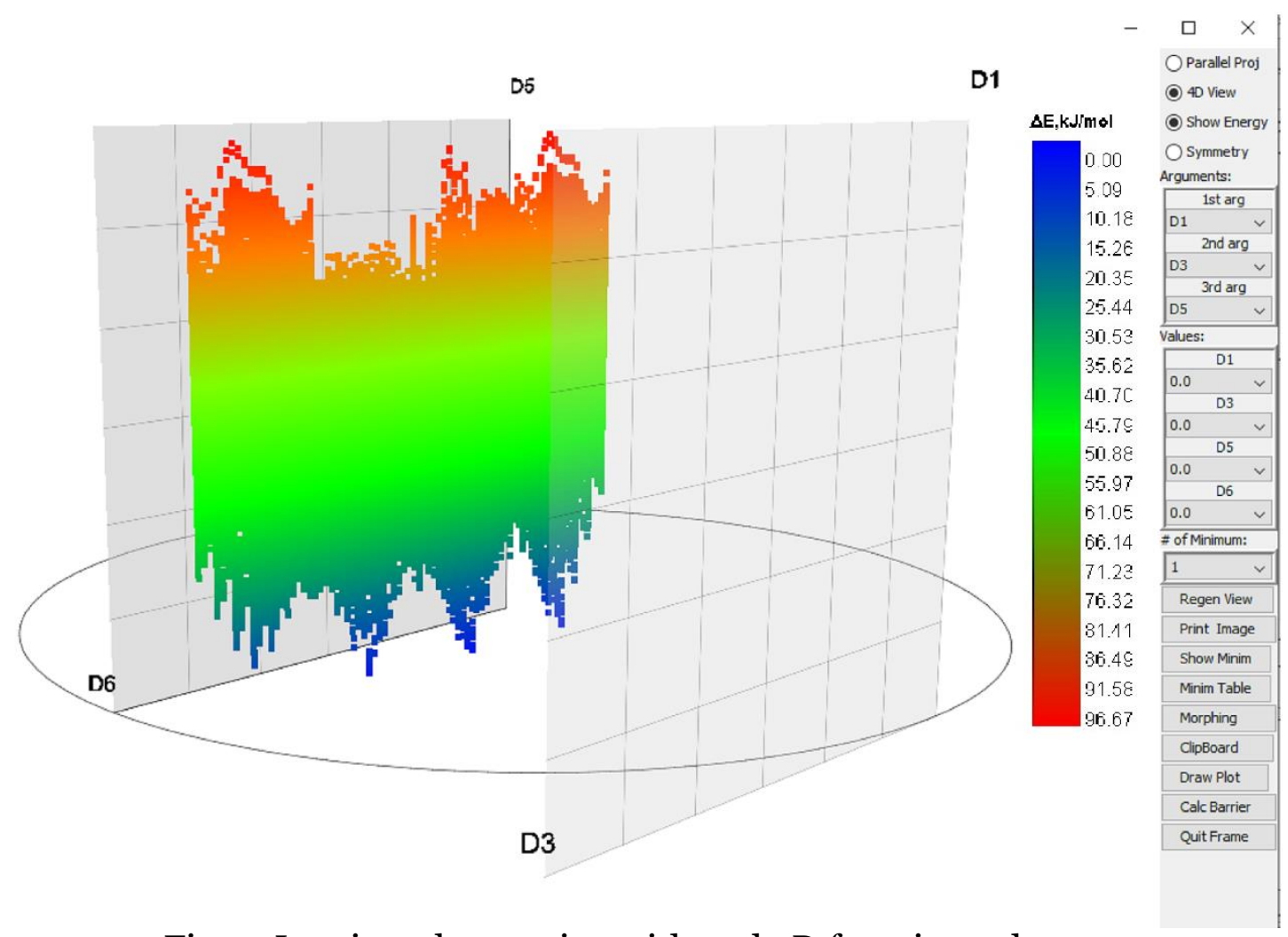

Fig. 9. Lumigraph mapping with real 5D function values

\section{Programming Tools}

When selecting a programming tool for development, we analyzed several platforms. After comparing the advantages and disadvantages of different platforms, the Java language [10] was chosen together with the Java 3D extension [11] which turned out to be the most suitable tool. Internet technologies, and in particular the Java language, have led to fundamental changes in the way applications are developed and deployed. The Java model "write once, run anywhere" reduces the complexity and cost typically associated with producing software on several different hardware platforms. With Java, the browser paradigm has become a convincing way to create applications for the Internet and corporate intranet. With the advent of new classes of applications for the web environment, the need for the full integration of multimedia capabilities in the browser paradigm is growing. Application developers require fewer high-level interfaces to work, but users prefer a seamless environment that is easy to use and maintain. In addition to software innovations, ASIC technology (specialized integrated circuit) and a high level of integration made hardware with support for accelerated three-dimensional visualization and multimedia available to users. We developed the ExDiGraph software package for the visualization of multidimensional surfaces together with their extrema on the basis of Java language [12]. The software allows visualizing multidimensional surfaces with dimensions up to $7 \mathrm{D}$.

\section{Conclusion}

The paper describes the method for visualization of a multidimensional hypersurface with its extrema by a Lumigraph model. The classical Lumigraph is not convenient enough for perception since it can be represented by a set of straight-line segments only, as described in this paper. The entire zones of the function extrema can hardly be seen on the whole, even in case Lumigraph is painted in different colors. An additional screen parallel to the Lumigraph planes contains a raster image of all zones of a multidimensional hypersurface. Additionally, such image is painted according to its color legend. We called this mechanism the mapping of a multidimensional surface onto an additional screen. Owing to such 
mechanism we can obtain more visually efficient raster image of the function together with all its extrema. This approach is applicable for visualization of a $5 \mathrm{D}$ hypersurface, depending on four variables. Further evaluation of this approach may be aimed at the increasing the dimension of the displayed hypersurface.

\section{Reference}

1. Wehrend S. and Lewis C. "A Problem-Oriented Classification of Visualization Techniques”, Proceedings of the 1st IEEE Conference on Visualization '90, 1990, pp.139143.

2. Etemadpour R. et al. Choosing Visualization Techniques for Multidimensional Data Projection Tasks: A Guideline with Examples. (2016, 166-186 pp 598. 166-186. 10.1007/978-3-319-29971-6_9.

3. Keim D. A. and Kriegel H.-P. "Visualization Techniques for Mining Large Databases: A Comparison", IEEE Transactions on Knowledge and Data Engineering, vol.8, no.6, 1996 pp.923-938.

4. Bondarev A.E. On visualization problems in a generalized computational experiment / Scientific Visualization, 2019, volume 11, number 2, pages 156 - 162, http://doi.org/ $10.26583 /$ Sv.11.2.12

5. Bondarev A.E., Galaktionov V.A., Shapiro L.Z. Processing and visual analysis of multidimensional data / Scientific Visualization. V.9, № 5, c.86-104, 2017, DOI: http://doi.org/10.26583/sv.9.5.08

6. E. Popov, A. Batiukov, N. Vogt, T. Popova, J. Vogt. Visualization and Analysis of Molecular Potential Energy Surface (Pes) and Its Minima. IADIS International Conference Interfaces and Human Computer Interaction 2019 (part of MCCSIS 2019), Porto, 2019. pp. 411-415.

7. Popov, E. V., Batiukov, A. A., Vogt, N., Popova, T. P., \& Vogt, J. (2020). Visualization and Minima Finding of Multidimensional Hypersurface. In P. Isaias, \& K. Blashki (Eds.), Interactivity and the Future of the Human-Computer Interface (pp. 282-309). Hershey, PA: IGI Global. doi:10.4018/978-1-7998-2637-8.cho15

8. B. Shneiderman, "The Eyes Have It: A Task by Data Type Taxonomy of Information Visualization," presented at IEEE Symposium on Visual Languages '96, Boulder, CO, 1996.

9. Götler S. et al, The Lumigraph. In Computer Graphics, Annual Conference Series (Proc. SIGGRAPH '96): 1996. pp. 43-54.

10. Niemeyer, P., \& Knudsen, J. (2013). Learning Java (4th ed.). O’Reilly Media, Inc.

11. Selman, D. (2003). Java3D Programming. Austin, TX: Manning Publications Co.

12. Popov E.V., Batiukov A.A. Exi Diastaseon Grafima (ExiDiaGraph). Certificate of a computer program RU 2020612284, 19/02/2020. Application No. 2020611368/69 of $10 / 02 / 2020$. 